\title{
NATALIYA CHEKHOVA
}

\section{PASCAL HUBERT}

\section{Ali MessaOudi}

\section{Propriétés combinatoires, ergodiques et arithmétiques} de la substitution de Tribonacci

Journal de Théorie des Nombres de Bordeaux, tome 13, nº 2 (2001), p. 371-394

<http://www.numdam.org/item?id=JTNB_2001_13_2_371_0>

(C) Université Bordeaux 1, 2001, tous droits réservés.

L'accès aux archives de la revue "Journal de Théorie des Nombres de Bordeaux » (http://jtnb.cedram.org/) implique l'accord avec les conditions générales d'utilisation (http://www.numdam.org/conditions). Toute utilisation commerciale ou impression systématique est constitutive d'une infraction pénale. Toute copie ou impression de ce fichier doit contenir la présente mention de copyright.

\section{Numdam}

Article numérisé dans le cadre du programme

Numérisation de documents anciens mathématiques

http://www.numdam.org/ 


\title{
Propriétés combinatoires, ergodiques et arithmétiques de la substitution de Tribonacci
}

\author{
par Nataliya CHEKHOVA, Pascal HUBERT \\ et ALI MESSAOUDI*
}

RÉSUMÉ. Nous étudions certaines propriétés combinatoires, ergodiques et arithmétiques du point fixe de la substitution de Tribonacci (introduite par G. Rauzy) et de la rotation du tore $\mathbb{T}^{2}$ qui lui est associée.

Nous établissons une généralisation géométrique du théorème des trois distances et donnons une formule explicite pour la fonction de récurrence du point fixe. Nous donnons des propriétés d'approximation diophantienne du vecteur de la rotation de $\mathbb{T}^{2}$ : nous montrons, que pour une norme adaptée, la suite de meilleure approximation de ce vecteur est la suite des nombres de Tribonacci. Nous calculons enfin les invariants ergodiques $F$ et $F_{C}$ du système dynamique associé à la substitution.

ABSTRACT. We study combinatoric, ergodic and arithmetic properties of the fixed point of Tribonacci substitution (first introduced by G. Rauzy) and of the related rotation of the two dimentional torus.

We give a geometric generalization of the three distances theorem and an explicit formula for the recurrence function of the fixed point of the substitution. We state Diophantine approximation's properties of the vector of the rotation of $\mathbb{T}^{2}$ : we prove that, for a suitable norm, the sequence of best approximation of this vector is the sequence of Tribonacci numbers. We compute the ergodic invariants $F$ and $F_{C}$ of the symbolic system related to the substitution.

\section{Introduction}

Les suites sturmiennes constituent une famille d'exemples particulièrement intéressants en dynamique symbolique et théorie des langages. On peut les définir, par exemple, comme les suites non ultimement périodiques, de complexité minimale. Elles ont fait l'objet de très nombreuses études,

Manuscrit reçu le 25 octobre 1999.

*Financé par une bourse du CNPq-Brazil, 150016 / 97-2. 
tant du point de vue combinatoire (voir par exemple [5], [6], [15], [20], [36], [40], [48]) que pour les interprétations géométriques (cf [4], [28], [47]), arithmétiques sous-jacentes (cf [32],[33], [34],[35], [42], [22]) et ergodiques ([3]). Les suites sturmiennes s'interprètent géométriquement comme les codages "canoniques" des rotations irrationnelles du tore $\mathbb{T}^{1}$.

Parmi les nombreuses généralisations des suites sturmiennnes, G. Rauzy (cf. [45]) a introduit un exemple de système symbolique qui constitue un "codage naturel" d'une rotation du tore $\mathbb{T}^{2}$. Le système symbolique est le système associé à la substitution de Tribonacci $\sigma$ définie par :

$$
\begin{aligned}
& \sigma(0)=01 \\
& \sigma(1)=02 \\
& \sigma(2)=0 .
\end{aligned}
$$

Du point de vue de la théorie ergodique, le résultat de G. Rauzy signifie que le système symbolique de Tribonacci est métriquement isomorphe à une rotation $R_{\alpha}$ de $\mathbb{T}^{2}$.

La rotation $R_{\alpha}$ de $\mathbb{T}^{2}$ s'obtient comme le quotient d'un échange de morceaux, dans le plan complexe, de domaine fractal (appelé fractal de Rauzy). Le fractal de Rauzy $\mathcal{E}$ possède diverses propriétés géométriques : c'est un compact de $\mathbb{C}$, connexe, à frontière fractale et à intérieur simplement connexe et il induit un pavage périodique de $\mathbb{C}$ modulo un réseau.

Le fractal de Rauzy a fait l'objet de plusieurs études (voir [2], [45], [30], [37], [38], [49]) et peut être relié à différents problèmes :

- Système de numération complexe [37], [38].

- Représentation géométrique des systèmes dynamiques symboliques [45], [30], [37], [38], [49], [50], [51].

- Méthode de Dekking pour la construction d'objets fractals [30].

- Pavages quasi-périodiques du plan [30].

- Partitions de Markov pour les automorphismes hyperboliques du tore $\mathbb{T}^{3}$ [2], [37].

Dans cet article, nous étudions certaines propriétés combinatoires et ergodiques du point fixe $u$ de la substitution de Tribonacci qui généralisent des propriétés connues pour les suites sturmiennes.

Nous donnons une généralisation du théorème des trois distances dû à Sós [52], qui a été étudié, récemment, d'un point de vue combinatoire par $\mathrm{P}$. Alessandri et V. Berthé (cf. [1]). Plus précisément, soit $\psi$ la conjugaison entre le système symbolique et la rotation $R_{\alpha}$ et $v$ un mot fini apparaissant dans $u$, on appelle $\psi([v])$, l'image du cylindre $[v]$ par l'application $\psi$; étant donné un nombre entier $n$, on note $\psi\left(L_{n}(u)\right)$ l'ensemble formé des $\psi([v])$ où $v$ est un mot de longueur $n$ du langage de $u$. Nous montrons que, pour tout entier $n$, l'ensemble $\psi\left(L_{n}(u)\right)$ est constitué, à translation près, de trois ou quatre sous-ensembles explicites du plan complexe. 
Nous donnons une formule explicite pour la fonction de récurrence $R$ de la suite de $u$, qui mesure la longueur minimale qu'il faut prendre dans la suite pour être certain de voir tous les mots de longueur $n$. On peut vérifier, par exemple, que :

$$
\limsup _{n \rightarrow+\infty} \frac{R(n)}{n}=10,605 \ldots,
$$

ce qui vérifie, dans ce cas particulier, la conjecture de Rauzy [46], selon laquelle cette quantité est toujours supérieure à $3,618 \ldots$, valeur atteinte pour la suite de Fibonacci. Cette conjecture a été récement résolue par J. Cassaigne [10].

Nous donnons des propriétés d'approximation simultanée du vecteur $\xi$ de la rotation $R_{\alpha}$. Nous remarquons que le vecteur $\xi$ est mal approché par les rationnels et nous montrons que, pour une norme adaptée, la suite de meilleure approximation du vecteur $\xi$ (voir définition 6) est la suite numérique de Tribonacci définie par la relation de récurrence :

$$
T_{0}=T_{1}=0, T_{2}=1, T_{n+3}=T_{n+2}+T_{n+1}+T_{n}, n \geq 0 .
$$

Nous calculons, enfin, les nombres de recouvrement $F_{C}$ et $F$, qui sont des invariants de conjugaison topologique du système dynamique symbolique associé à la suite de Tribonacci ; ils mesurent, respectivement, la proportion de la suite qui peut être recouverte par des apparitions d'un seul mot dont toutes les apparitions sont sans chevauchement, et la proportion de la suite qui peut être recouverte par des apparitions sans chevauchement d'un seul mot, voir les définitions 7,8 , ci-après. Ces invariants ont été calculés par N. Chekhova pour les suites sturmiennes (cf [12]).

Ces résultats symboliques ont une interprétation géométrique, sous forme de propriétés ergodiques nouvelles de la rotation $R_{\alpha}$ : l'invariant $F_{C}$ que nous avons calculé se traduit par l'existence de tours de Rokhlin constituées d'ensembles simplement connexes, une tour recouvrant $0,618 \ldots$ de l'espace et trois tours recouvrant tout l'espace, ce qui améliore, dans ce cas particulier, certains résultats de Chevallier (cf [14]).

\section{Préliminaires}

2.1. Dynamique symbolique et langages formels. Soit $\Lambda$ un alphabet fini ; on considère les suites unilatérales $\left(x_{0}, \cdots, x_{n}, \cdots\right), x_{n} \in \Lambda$.

Un mot de longueur $l(w)=h$ est une suite finie $w=w_{1} \cdots w_{h}$ d'éléments de $\Lambda$. La concaténation de deux mots $v$ et $w$ se note $v w$. Le mot $w=w_{1} \cdots w_{h}$ apparaît au terme $i$ dans une suite $\left(x_{n}\right)$ ou dans un mot $x_{0} \cdots x_{s}$ si $x_{i}=w_{1}$, $\ldots, x_{i+h-1}=w_{h}$.

Soit $z$ appartenant à $\Lambda^{\mathbb{N}}$, le langage de longueur $h$, noté $L_{h}(z)$ est l'ensemble des mots de longueur $h$ apparaissant dans $z$; le langage de $z$ est l'ensemble des mots apparaissant dans $z$. 
On définit le décalage $T$ sur l'espace $\Lambda^{\mathbb{N}}$, pour $x=\left(x_{n}, n \in \mathbb{N}\right)$, par $(T x)_{n}=$ $x_{n+1}$. Pour une suite $z$, on définit l'ensemble $X_{z}$ comme l'adhérence dans $\Lambda^{\mathbb{N}}$ muni de la topologie discrète de l'ensemble $\left(T^{n} z, n \in \mathbb{N}\right)$.

Un système dynamique symbolique est le système dynamique topologique $\left(X_{z}, T\right)$ pour une suite $z$ sur un alphabet $\Lambda$. Le système dynamique symbolique qu'on considère ici est minimal (toute orbite est dense) et uniquement ergodique : il y a une seule mesure de probabilité invariante par $T$; on peut alors définir des fréquences.

La fréquence $f(w)$ d'un mot est $\lim _{n \rightarrow+\infty} \frac{N_{n}(w)}{n+1}$, où $N_{n}(w)$ est le nombre d'apparitions de $w$ dans $z_{0} \cdots z_{n}$.

Dans un système dynamique symbolique, étant donné un mot $w$, on appelle cylindre associé à $w$, noté $[w]$, l'ensemble des $x$ de $X_{z}$ tel que $w$ apparaît dans $x$ au terme 0 ; la hauteur du cylindre $[w]$ est la longueur de $w$. Soit $w$ un élément de $L_{n}(z)$ et $f(w)$ la fréquence d'apparition du mot $v$ dans $z$, il est connu que $f(w)$ est égale à la mesure du cylindre [w] (voir [37], [38]).

\subsection{La substitution de Tribonacci.}

Définition 1. Soit $A$ l'ensemble $\{0,1,2\}$, nous appelons substitution de Tribonacci l'application $\sigma$ de $A$ dans $A^{*}$ définie par :

$$
\begin{aligned}
& \sigma(0)=01 \\
& \sigma(1)=02 \\
& \sigma(2)=0 .
\end{aligned}
$$

Nous étendons $\sigma$ en une application de $A^{\mathbb{N}}$ dans $A^{\mathbb{N}}$ par concaténation, c'est-à-dire, pour tout $\left(a_{n}\right)_{n} \in A^{\mathbb{N}}$,

$$
\sigma\left(a_{0} a_{1} \cdots a_{n} \cdots\right)=\sigma\left(a_{0}\right) \sigma\left(a_{1}\right) \cdots \sigma\left(a_{n}\right) \cdots
$$

$\sigma$ est contractante pour la métrique naturelle sur $A^{\mathbb{N}}$, elle a donc un seul point fixe que l'on notera $u=\left(u_{n}\right)_{n \in \mathbb{N}}$.

A l'instar de la pratique adoptée dans le cas de Fibonacci (substitution définie par $0 \rightarrow 01,1 \rightarrow 0$ ), nous appelons suite de Tribonacci [9] la suite $u_{0} u_{1} \cdots$. On remarque que la longueur du mot $\sigma^{n} 0$ est égale à $T_{n+3}$, où $T_{n}$ est le nombre de Tribonacci défini par la relation de récurrence :

$$
T_{0}=T_{1}=0, T_{2}=1, T_{n+3}=T_{n+2}+T_{n+1}+T_{n}, n \geq 0 .
$$

On est amené à considérer le polynôme $P(x)=x^{3}-x^{2}-x-1$. Ce polynôme a une racine réelle $\beta=1,83929 \ldots$ strictement supérieure à 1 et deux racines complexes conjuguées $\alpha$ et $\bar{\alpha}$ de module inférieur strictement à 1 , on a donc $\beta|\alpha|^{2}=1$. (Dans tout ce qui suit, $\alpha$ est le conjugué de $\beta$ de partie imaginaire négative, cette convention est arbitraire et n'a aucune influence sur les résultats ci-dessous ; $\alpha \approx-0.419-0.606 i$.)

On considère désormais le système dynamique symbolique $\left(X_{u}, T\right)$ associé à $u$; il est minimal et uniquement ergodique car la substitution est primitive [44]. 


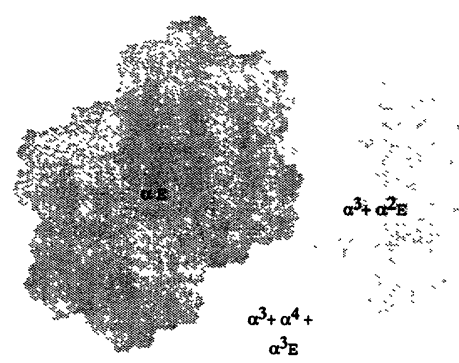

Le fractal de Rauzy : $E$

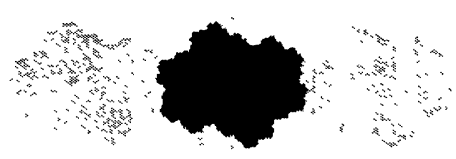

Pavage périodique du plan par le fractal

de Rauzy

Figure 1. Fractal de Rauzy

\subsection{Fractal de Rauzy.}

Définition 2. Soit $\varepsilon=\left(\varepsilon_{i}\right)_{i \in \mathbb{N}}$ une suite à valeurs dans $\{0,1\}$. On dit que $\varepsilon$ est une suite admissible si, pour tout entier naturel $i$, elle vérifie $\varepsilon_{i} \varepsilon_{i+1} \varepsilon_{i+2}=0$. On note $\mathcal{N}_{f}$ l'ensemble des suites admissibles.

Remarque. Une suite finie $\varepsilon$ à valeurs dans $\{0,1\}$ est admissible si la suite infinie obtenue en prolongeant $\varepsilon$ à gauche par une infinité de 0 est admissible.

Définition 3. Le fractal de Rauzy est l'ensemble (voir figure 2.3)

$$
\mathcal{E}=\left\{\sum_{i=3}^{+\infty} \varepsilon_{i} \alpha^{i} \text { où }\left(\varepsilon_{i}\right)_{i \geq 3} \in \mathcal{N}_{f}\right\}
$$

Le fractal de Rauzy est partagé en trois régions similaires qui induisent un autre pavage non périodique et auto-similaire du plan complexe. Ces régions sont : $\mathcal{E}_{0}=\alpha \mathcal{E}, \mathcal{E}_{1}=\alpha^{3}+\alpha^{2} \mathcal{E}$ et $\mathcal{E}_{2}=\alpha^{3}+\alpha^{4}+\alpha^{3} \mathcal{E}$.

Conjugaison entre le système symbolique et la rotation. Soit $R_{\alpha}$ la rotation (échange de trois morceaux) sur $\bigcup_{i=0,1,2} \operatorname{int}\left(\mathcal{E}_{i}\right)$ définie par $R_{\alpha}(z)=$ 
$z+\alpha^{2}$ modulo $(\mathbb{Z}+\mathbb{Z} \alpha)$ et soit $\lambda$ la mesure de Lebesgue bidimensionnelle. Le théorème suivant établit le lien entre substitution de Tribonacci et fractal de Rauzy (voir [45], [37], [38]).

Théorème 1 (Rauzy). Soit $m$ l'unique mesure de probabilité invariante pour le système symbolique $\left(X_{u}, T\right)$. Le système dynamique $\left(X_{u}, T, m\right)$ est métriquement isomorphe au système dynamique $\left(\mathcal{E}, R_{\alpha}, \lambda\right)$ par une application $\psi$. En outre $\psi$ est continue, surjective et injective sur $\operatorname{Orb}(u)$, et pour tout $x \in \Omega, \psi(\sigma(x))=\alpha \psi(x)$.

Remarque. Pour une définition explicite de $\psi$, on peut voir [38] page 149.

\section{Graphe de Rauzy}

Définition 4. Pour une suite $u$ et un entier $h$, on définit le graphe de Rauzy (ou graphe des mots de longueur $h$ ) $\Gamma_{h}$ de la manière suivante : les sommets sont les points de $L_{h}(u)$, et il y a une arête de $w$ vers $w^{\prime}$ si $w$ et $w^{\prime}$ apparaissent successivement dans $u$, c'est-à-dire si $w=a v$ et $w^{\prime}=v b$ pour des lettres $a$ et $b$ et un mot $v$ de $L_{h-1}(u)$; on étiquette cette arête par $a v b \in L_{h+1}(u)$, et l'ensemble des arêtes est $L_{h+1}(u)$.

Nous étudions ci-dessus la forme des graphes de Rauzy pour la suite de Tribonacci $u$. Pour tout nombre entier $h$, le graphe $\Gamma_{h}$ comporte un point unique $D_{h}$ triprolongeable à droite (trois arêtes $D_{h} 0, D_{h} 1$ et $D_{h} 2$ le quittent) et un point unique $G_{h}$ triprolongeable à gauche (trois arêtes $0 G_{h}$, $1 G_{h}$ et $2 G_{h}$ y arrivent) ; $D_{h}$ et $G_{h}$ peuvent être confondus. Pour tout autre point, il y a une seule arête entrante une seule arête sortante. En conséquence, la suite de Tribonacci est une suite d'Arnoux-Rauzy et son graphe de Rauzy obéit aux règles définies dans [4], que nous explicitons ci-après :

On partitionne les sommets et les arêtes de $\Gamma_{h}$ en quatre branches; une branche est un ensemble de sommets et d'arêtes, et sa longueur est le nombre de sommets qu'elle contient.

- La branche centrale comprend le sommet $G_{h}$, l'unique arête sortante de $G_{h}$, le sommet vers lequel va cette arête, l'unique arête sortante de ce sommet, et ainsi de suite jusqu'au sommet $D_{h}$ inclus ; si $D_{h}=G_{h}$, la branche centrale est réduite au sommet $D_{h}=G_{h}$.

- pour $i=0,1,2$, la branche $i$ comprend l'arête $D_{h} i$, le sommet vers lequel va cette arête, l'unique arête sortante de ce sommet, et ainsi de suite jusqu'au sommet $G_{h}$ exclu ; si l'arête $D_{h} i$ va vers $G_{h}$, la branche $i$ est réduite à l'arête $D_{h} i$.

Nous verrons que, sauf pour $h=1$, les longueurs de ces trois dernières branches sont toujours différentes, et les appellerons respectivement branche courte, branche moyenne et branche longue. Le circuit court $C_{h}$ (resp. 

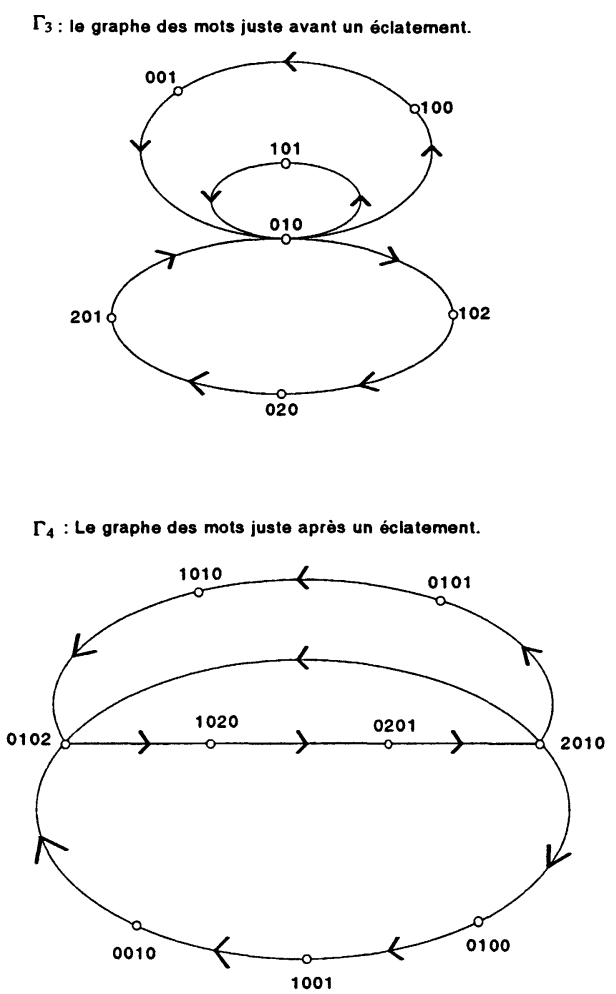

FiguRE 2. Graphes de Rauzy

moyen $M_{h}$ et long $L_{h}$ ) part de $G_{h}$ et est composé de la branche centrale puis de la branche courte (resp. moyenne et longue).

Les sommets de $\Gamma_{h+1}$ sont les arêtes de $\Gamma_{h} ;$ si $D_{h} \neq G_{h}$, les sommets d'une branche de $\Gamma_{h+1}$ sont les arêtes de la même branche de $\Gamma_{h}$ (il y a fente d'une arête). De ce fait, la longueur de la branche centrale diminue de 1 quand on passe de $h$ à $h+1$.

Donc, pour une infinité de $h$, la branche centrale de $\Gamma_{h}$ est de longueur 1 , par conséquent réduite à un sommet; d'où $G_{h}=D_{h}$ : on dit qu'il y a un éclatement.

Les différents types d'éclatements sont décrits dans [4], mais nous introduisons ici une nouvelle terminologie ; nous disons qu'il y a un éclatement renversant (noté $E_{L}$ ) si les sommets de la branche centrale de $\Gamma_{h+1}$ sont les arêtes de la branche longue de $\Gamma_{h}$; il y a un éclatement moyen $\left(E_{M}\right)$ si les sommets de la branche centrale de $\Gamma_{h+1}$ sont les arêtes de la branche moyenne de $\Gamma_{h}$; il y a un éclatement court $\left(E_{C}\right)$ si les sommets de la branche centrale de $\Gamma_{h+1}$ sont les arêtes de la branche courte de $\Gamma_{h}$; dans les trois cas, la branche courte de $\Gamma_{h+1}$ est réduite à une arête (voir figure 3). 
Si l'on énumère successivement les mots de longueur $h$ apparaissant dans la suite $u$ au terme $i$ pour $i=0, \ldots n, \ldots$, on obtient un chemin infini $\gamma_{h}$ dans le graphe $\Gamma_{h}$, composé d'une succession de circuits courts, moyens et longs (le premier étant éventuellement tronqué). Si en $h$ il y a fente, $\gamma_{h}$ se déduit de $\gamma_{h+1}$ en remplaçant $C_{h+1}$ par $C_{h}, M_{h+1}$ par $M_{h}$ et $L_{h+1}$ par $L_{h}$; s'il y a éclatement court, $\gamma_{h}$ se déduit de $\gamma_{h+1}$ en remplaçant $C_{h+1}$ par $C_{h}, M_{h+1}$ par $C_{h} M_{h}$ et $L_{h+1}$ par $C_{h} L_{h} ;$ s'il y a éclatement moyen, $\gamma_{h}$ se déduit de $\gamma_{h+1}$ en remplaçant $C_{h+1}$ par $M_{h}, M_{h+1}$ par $M_{h} C_{h}$ et $L_{h+1}$ par $M_{h} L_{h} ;$ s'il y a éclatement renversant, $\gamma_{h}$ se déduit de $\gamma_{h+1}$ en remplaçant $C_{h+1} \operatorname{par} L_{h}, M_{h+1} \operatorname{par} L_{h} C_{h}$ et $L_{h+1} \operatorname{par} L_{h} M_{h}$.

Dans toute la suite, si, par abus de langage, nous parlons d'un mot de la branche centrale (resp. courte, moyenne, longue), il s'agira toujours d'un sommet de cette branche. On appelle longueur d'une branche son nombre de sommets.

La méthode de [7] permet de calculer de proche en proche les longueurs des circuits et les fréquences des mots de chaque branche ; le résultat suivant est à rapprocher du résultat de [17] sur la suite de Fibonacci.

Proposition 1. Pour une valeur de h située entre le $n$-2-ème éclatement (non compris) et le n-1-ème éclatement (compris), les longueurs des circuits sont

$$
\left|L_{h}\right|=T_{n+2}, \quad\left|M_{h}\right|=T_{n}+T_{n+1}, \quad\left|C_{h}\right|=T_{n+1} .
$$

Tous les mots d'une même branche ont la même fréquence. Les branches, centrale, moyenne et longue, contiennent toujours des sommets, la branche courte en contient si et seulement s'il y a fente pour $h-1$; donc il y a au plus trois ou quatre fréquences différentes. Les fréquences communes respectives des mots de la branche longue, moyenne, courte et centrale sont

$$
f_{L}=\frac{1}{\beta^{n}}, \quad f_{M}=\frac{1}{\beta^{n+1}}, \quad f_{C}=\frac{1}{\beta^{n+2}}, \quad f_{+}=f_{L}+f_{M}+f_{C}=\frac{1}{\beta^{n-1}} .
$$

Preuve. Nous montrons d'abord que tous les éclatements sont renversants ; pour cela, nous montrons par récurrence sur $n$ que pour un $h$ situé juste avant le $n$-ème éclatement la suite $\gamma_{h}$ est la suite de Tribonacci elle-même, où on a remplacé $0 \operatorname{par} L_{h}, 1$ par $M_{h}$ et $2 \operatorname{par} C_{h}$. C'est vrai pour $n=1$ en choisissant pour $L_{1}$ le circuit comprenant les sommets 0 et 1 , pour $M_{1}$ les sommets 0 et 2 , pour $C_{1}$ le sommet 0 , car la décomposition de la suite de Tribonacci en mots $01,02,0$ est unique. Si c'est vrai pour $n$, soit $h$ juste avant le $n$-ème éclatement ; comme $\gamma_{h}$ est invariante par $\sigma$ on peut écrire les mots de longueur $h+1$ apparaissant dans la suite $u$ au terme $i$ pour $i=0, \ldots n, \ldots$, en tant qu'arêtes de $\Gamma_{h}$ ou sommets de $\Gamma_{h+1}$, comme un chemin infini qui est une concaténation de circuits $L_{h}, L_{h} C_{h}$ et $L_{h} M_{h}$; donc ce chemin ne peut être une concaténation de $M_{h}, M_{h} C_{h}$ et $M_{h} L_{h}$ ou de $C_{h}, C_{h} M_{h}$ et $C_{h} L_{h}$; donc l'éclatement est renversant; donc la suite 
$\gamma_{h+1}$ s'obtient à partir de $\gamma_{h}$ en remplaçant $L_{h}$ par $L_{h+1}, L_{h} C_{h}$ par $M_{h+1}$, $L_{h} M_{h}$ par $L_{h+1}$, et l'hypothèse de récurrence est vérifiée avant le $n+1$-ème éclatement.

Les longueurs des circuits se calculent donc grâce à l'évolution des graphes indiquée ci-dessus : pour $h=1$, on a

$$
\left|C_{1}\right|=1, \quad\left|L_{1}\right|=\left|M_{1}\right|=2 ;
$$

si, pour $h$, il y a fente, on a

$$
\left|C_{h+1}\right|=\left|C_{h}\right|, \quad\left|M_{h+1}\right|=\left|M_{h}\right|, \quad\left|L_{h+1}\right|=\left|L_{h}\right| ;
$$

si, pour $h$, il y a éclatement, on a

$$
\begin{aligned}
\left|C_{h+1}\right| & =\left|L_{h}\right|, \\
\left|M_{h+1}\right| & =\left|L_{h}\right|+\left|C_{h}\right|, \\
\left|L_{h+1}\right| & =\left|L_{h}\right|+\left|M_{h}\right| .
\end{aligned}
$$

Soit $V$ un élément de $L_{h}(u)$ et $f(V)$ sa fréquence, supposons, par exemple, que $V$ appartienne à la branche courte, soit $W$ l'élément de la branche courte tel que $V=x Y$ et $W=Y z$ où $x$ et $z$ sont dans $\{0,1,2\}$. Comme $V$ est prolongeable d'une façon unique à droite et à gauche, nous avons $f(V)=f(V z)=f(Y z)$. Par conséquent tous les mots de la branche courte (respectivement moyenne, longue) ont la même fréquence $f_{C}$ (resp $f_{M}$ et $f_{L}$ ). De même, les mots de la branche centrale ont la même fréquence $f_{+}$. Par ailleurs, pour tout entier naturel $h$ non nul, nous avons

$$
f\left(D_{h-1}\right)=f\left(D_{h-1} 0\right)+f\left(D_{h-1} 1\right)+f\left(D_{h-1} 2\right) .
$$

Il faut étudier deux cas :

Premier cas : Si $D_{h-1} \neq G_{h-1}$ (il y a fente pour $h-1$ ), $D_{h-1}$ a un seul prolongement à gauche, d'où

$$
f\left(D_{h}\right)=f\left(D_{h-1}\right)=f\left(D_{h-1} 0\right)+f\left(D_{h-1} 1\right)+f\left(D_{h-1} 2\right) .
$$

Il en découle que $f_{+}=f_{C}+f_{M}+f_{L}$. Dans ce cas, chaque branche contient des sommets, et l'ensemble $\left\{f(v) \mid v \in L_{h}(u)\right\}$ prend au plus quatre valeurs.

Deuxième cas : Si $D_{h-1}=G_{h-1}$, alors il existe $x$ et $y$ dans $\{0,1,2\}$ tels que $G_{h}=G_{h-1} x$ et $D_{h}=y D_{h-1}=y G_{h-1}$. Dans ce cas, le graphe $\Gamma_{h}$ est en fente et la branche courte est réduite à une arête. Il y a donc, au plus, trois fréquences, qui sont $f_{+}, f_{L}$ et $f_{M}$. On continuera toutefois à définir $f_{C}$ par $f_{C}=f_{+}-f_{L}-f_{M}$, bien qu'à ce stade ce ne soit la fréquence d'aucun mot.

Nous allons maintenant calculer ces fréquences par récurrence ; si $h=1$ alors $f(0)=\frac{1}{\beta}, f(1)=\frac{1}{\beta^{2}}$ et $f(2)=\frac{1}{\beta^{3}}$ (voir [37]). Le circuit court est réduit à 0 , le circuit moyen comprend 0 et 2 , le circuit long comprend 0 et 
1 (le choix du circuit moyen et du circuit long n'est pas ambigu en raison de la suite du processus, qui impose $f_{L}>f_{M}$ ), et on a

$$
f_{+}=f(0), \quad f_{L}=f(1), \quad f_{M}=f(2) .
$$

On définit $f_{C}$, sans objet à ce stade, puisqu'il n'y a pas de sommet sur la branche courte, par $f_{C}=f_{+}-f_{L}-f_{M}$.

Ensuite, le raisonnement ci-dessus, ou celui de [7], montrent que les fréquences sont inchangées par une fente (dans le cas de la première fente après un éclatement, la fréquence $f_{C}$ qui n'est fréquence d'aucun mot de $\Gamma_{h}$ devient la fréquence du mot de la branche courte de $\Gamma_{h+1}$ ), tandis que, s'il y a éclatement, on a

$$
\begin{aligned}
f_{C}^{\prime}+f_{M}^{\prime}+f_{L}^{\prime} & =f_{L} \\
f_{M}^{\prime} & =f_{C} \\
f_{L}^{\prime} & =f_{M},
\end{aligned}
$$

où $f_{C}, f_{L}, f_{M}$ sont les fréquences avant l'éclatement et $f_{C}^{\prime}, f_{L}^{\prime}$ et $f_{M}^{\prime}$ les fréquences après l'éclatement (juste après l'éclatement, $f_{C}^{\prime}$ n'est la fréquence d'aucun mot mais reste définie par $f_{C}^{\prime}=f_{+}^{\prime}-f_{L}^{\prime}-f_{M}^{\prime}$ ). On poursuit ainsi la récurrence.

\section{Généralisation du théorème des trois distances}

Le théorème ci-dessous est une généralisation géométrique du théorème des trois distances.

Théorème 2. Soit $n$ un entier naturel non nul, l'ensemble $\{\psi([v]) \mid v \in$ $\left.L_{n}(u)\right\}$ est constitué de trois ou quatres régions à translations près qui sont de la forme $\alpha^{k} \mathcal{E}, \alpha^{k+1} \mathcal{E}$ et $\alpha^{k+2} \mathcal{E}$ ou bien $\alpha^{k} \mathcal{E}, \alpha^{k+1} \mathcal{E}, \alpha^{k+2} \mathcal{E}$ et $\alpha^{k+3} \mathcal{E}$, suivant les valeurs de $n$.

Preuve. Montrons que si $f(v)=f(w)$, $v$ et $w$ dans $L_{n}(u)$, alors les régions $\psi([v])$ et $\psi([w])$ sont similaires au fractal de Rauzy et sont translatées l'une de l'autre.

Considérons les fonctions de $\mathbb{C}$ dans $\mathbb{C}$ définies par $\psi_{0}: z \mapsto \alpha z, \psi_{1}$ : $z \mapsto \alpha^{3}+\alpha^{2} z$ et $\psi_{2}: z \mapsto \alpha^{3}+\alpha^{4}+\alpha^{3} z$. Nous avons, par construction, $\psi_{i}(\mathcal{E})=\psi([i])$ pour tout $i \in\{0,1,2\}$; plus généralement, en vertu du Théorème 1 , nous avons le résultat suivant (voir [38] page 151).

Lemme 1. Soit $v_{1} \cdots v_{n}$ un élément de $L_{n}(u)$. Il existe $i_{1}, \ldots, i_{r}$ des éléments de $\{0,1,2\}$ où $r<n$, tels que $\psi\left(\left[v_{1} \cdots v_{n}\right]\right)=\psi_{i_{1}} \circ \cdots \circ \psi_{i_{r}}(\mathcal{E})$. Si, de plus, $v_{1} \cdots v_{n}$ est tri-prolongeable à droite, alors pour tout $j \in\{0,1,2\}$, on $a$

$$
\psi\left(\left[v_{1} \cdots v_{n} j\right]\right)=\psi_{i_{1}} \circ \cdots \circ \psi_{i_{r}} \circ \psi_{n_{j}}(\mathcal{E}),
$$

où $n_{j} \in\{0,1,2\}$ et $n_{j} \neq n_{i}$ si $i \neq j$.

Un corollaire immédiat du Lemme 1 est le suivant. 
Corollaire 1. Pour tout $v$ dans $L_{n}(u)$, il existe une suite $\left(\varepsilon_{i}\right)_{3 \leq i \leq N} \in \mathcal{N}_{f}$ et un entier $k>N$ tels que

$$
\psi([v])=\left(\sum_{i=3}^{N} \varepsilon_{i} \alpha^{i}\right)+\alpha^{k} \mathcal{E} .
$$

Le théorème 2 résulte alors de la Proposition 1 , du Corollaire 1 et du fait que $f(v)$ est proportionnel à la mesure de Lebesgue de $\psi([v])([38])$.

\section{Calcul exact de la fonction de récurrence}

Nous calculons, dans ce paragraphe, la fonction de récurrence $R$ de la suite $u$. Etant donné que $u$ est le point fixe d'une substitution primitive, il était déjà connu (voir [44]) qu'il existe une constante $L$ telle que, pour tout entier $n, R(n) \leq L n$. L'intérêt de notre résultat est de donner une formule explicite pour $R(n)$.

Définition 5. La fonction de récurrence d'une suite $z$ est la fonction, notée $R(n)$, qui à un entier $n$ associe le plus petit entier (fini ou non) $r$ tel que tout élément de $L_{n}(z)$ apparaisse dans $L_{r}(z)$.

On appelle temps de retour, noté $l(w)$, d'un mot $w$ dans $z$, la distance maximale entre deux occurrences consécutives de $w$ dans $z$, et on note $l(n)$ le maximum des $l(w)$ pour tous les mots $w \in L_{n}(z)$.

On appelle mot singulier de $z$ tout mot $w=x v y$ apparaissant dans $z$, tel que $x$ et $y$ soient des lettres et qu'il existe $x^{\prime} \neq x, y^{\prime} \neq y$ tels que $x^{\prime} v y$ et $x v y^{\prime}$ apparaissent dans $z$.

Lemme 2. Les fonctions $R$ et $l$ sont liées par

$$
\forall h \in \mathbb{N}^{*}, R(h)=l(h)+h-1 .
$$

Pour $h \geq 1$, le temps de retour maximal est donné par

$$
l(h)=\max _{w \in S_{h}(z)} l(w),
$$

où $S_{h}(z)$ est l'ensemble des mots singuliers de $z$ de longueur inférieure ou égale à $h$.

L'article [9] précise une méthode générale, essentiellement due à Hedlund et Morse [28], pour calculer la fonction de récurrence d'une suite. Le Lemme 2 est démontré dans [9, Propositions 2 et 3$]$.

Lemme 3. Dans le cas de la suite de Tribonacci, pour tout $n \geq 3$, il existe un unique mot singulier dont la longueur est située entre le $n-3$-ème et le $n$-2-ème éclatement ; il a pour longueur $g_{n}=\frac{T_{n+1}+T_{n-1}+1}{2}$ et pour temps de retour

$$
l(w)=l\left(g_{n}\right)=T_{n+4} .
$$


Preuve. Par définition, $w$ est un mot singulier de longueur $h$ si et seulement s'il est l'unique arête d'une branche de $\Gamma_{h-1}$, ce qui ne peut se produire que juste après un éclatement.

On se place au $n-3$-ème éclatement. Dans ce cas, la branche centrale est réduite à un seul sommet et la longueur correspondante $h$ est égale à $\frac{|L|+|M|+|C|-3}{2}=\frac{T_{n+1}+T_{n-1}-3}{2}$, en vertu de la Proposition 1 et du fait que la complexité $p$ de la suite $u$ vérifie, pour tout entier $h, p(h)=2 h+1$.

Après cet éclatement la nouvelle branche courte apparaît et il n'y a pas de sommets sur cette branche, son unique arète étant le mot singulier $w$. Le mot singulier $w$ apparaît à l'étape suivante, $g_{n}=\frac{T_{n+1}+T_{n-1}+1}{2}$, comme sommet dans le circuit court $C$, et n'apparait dans aucun des autres circuits, $L$ et $M$. Aux étapes suivantes, les circuits restent les mêmes jusqu'à l'éclatement suivant, puis sont remplacés, au fil des éclatements ultérieurs, par des concaténations de ces circuits, représentées par le schéma suivant (1) :

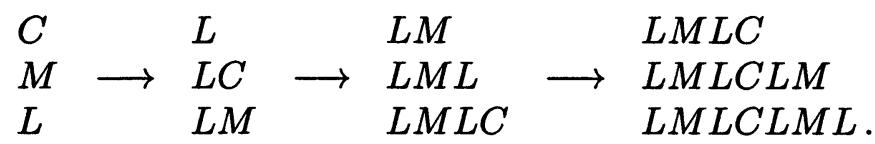

Le circuit $C$ (et donc le sommet $w$ ) apparaît dans chacun des trois chemins $L M L C, L M L C L M, L M L C L M L$. Comme la suite $u$ est un enchaînement de ces trois chemins, la distance maximale entre deux apparitions de $w$ est réalisée dans le chemin $L M L C L M L \mid L M L C$, qui est effectivement autorisé quand on passe à un éclatement de plus. Donc

$$
\begin{aligned}
l(w) & =4|L|+2|M|+|C|=4 T_{n+1}+2\left(T_{n}+T_{n-1}\right)+T_{n} \\
& =T_{n+4} .
\end{aligned}
$$

Les deux lemmes précédents entraînent immédiatement le résultat suivant.

Proposition 2. La fonction de récurrence de la suite de Tribonacci est donnée par

$$
R(h)=h-1+T_{n+4}
$$

pour

$$
\frac{T_{n+1}+T_{n-1}+1}{2} \leq h<\frac{T_{n+2}+T_{n}+1}{2}, \quad n \geq 1 .
$$

En particulier,

$$
\begin{aligned}
& \limsup _{n \rightarrow+\infty} \frac{R(n)}{n}=9+\frac{6 \beta-4}{\beta^{2}+1}=10,605 \ldots \\
& \liminf _{n \rightarrow+\infty} \frac{R(n)}{n}=5+\frac{4 \beta-2}{\beta^{2}+1}=6,222 \ldots
\end{aligned}
$$




\section{Approximation diophantienne}

Soit \|\|$_{0}$ une norme sur $\mathbb{R}^{2} ;$ pour $q \in \mathbb{N}, x \in \mathbb{R}^{2}$ fixés, on note $\|q x\|_{0}$ la distance de $q x$ au point entier le plus proche c'est-à-dire

$$
\|q x\|_{0}=\operatorname{Min}\left\{\left\|q x-\left(\begin{array}{c}
p \\
r
\end{array}\right)\right\|_{0}, \quad(p, r) \in \mathbb{Z}^{2}\right\} .
$$

On rappelle que $\left(T_{n}\right)$ est la suite des nombres de Tribonacci, $\beta$ la racine réelle de module strictement supérieur à un de $x^{3}=x^{2}+x+1, \alpha, \bar{\alpha}$ les deux racines complexes conjuguées ; on note $\xi=\left(\begin{array}{c}\theta \\ \theta^{2}\end{array}\right)$ où $\theta=\frac{1}{\beta}$.

Pour tout $N$, posons $\delta(N)=N \xi-\left(\begin{array}{l}\left|u_{N}\right|_{1} \\ \left|u_{N}\right|_{2}\end{array}\right)$ où $\left|u_{N}\right|_{1}$ (resp. $\left.\left|u_{N}\right|_{2}\right)$ est le nombre de 1 (resp. de 2) dans $u_{0} \cdots u_{N-1}$. La matrice $B=\left(\begin{array}{cc}-\theta & -\theta \\ 1-\theta^{2} & -\theta^{2}\end{array}\right)$ est semblable à $\left(\begin{array}{cc}\alpha & 0 \\ 0 & \bar{\alpha}\end{array}\right)$, et vérifie, (voir [45]), si $N=\sum_{n \geq 0} \epsilon_{n} T_{n+3}$, alors

$$
\delta(N)=\sum_{n \geq 0} \epsilon_{n} B^{n} \delta(1),
$$

où le vecteur $\delta(1)=\left(\begin{array}{c}\theta-1 \\ \theta^{2}\end{array}\right)$.

Nous appelons norme de Rauzy la norme \|\| , telle que, pour tout $v \in$ $\mathbb{R}^{2}$,

$$
\|B v\|=|\alpha|\|v\| .
$$

\section{Remarques.}

1) $\|\delta(1)\|=|\alpha|^{4}$ et $\left\|\delta\left(T_{n+3}\right)\right\|=|\alpha|^{n+4}$.

2) Il existe deux constantes $c_{0}$ et $c_{1}$ telles que, pour tout $n \in \mathbb{N}$,

$$
c_{0} \leq \frac{T_{n+3}}{\beta^{n}} \leq c_{1} \text {. }
$$

Théorème 3 (Rauzy [45]). Il existe un nombre strictement positif $c$ tel que, pour tout entier naturel non nul $q$, pour tout entier naturel $m$,

$$
\||| q \xi\| \mid<\theta^{\frac{m}{2}} \quad \text { implique } \quad \epsilon_{i}=0 \quad \forall i<m,
$$

où $q=\sum_{i \geq 0} \epsilon_{i} T_{i+3}$ et pour tout $i, \epsilon_{i} \epsilon_{i+1} \epsilon_{i+2}=0$.

Proposition 3. Il existe $K$ tel que pour tout $q$

$$
q^{\frac{1}{2}}\||q \xi|\| \geq K \text {. }
$$

Preuve. D'après le théorème ci-dessus, $\left\||q \xi \||<c \theta^{\frac{m}{2}}\right.$ implique $q \geq T_{m+3}$. En particulier $\||q \xi|\|<c c_{0}^{\frac{1}{2}} T_{m+3}^{-\frac{1}{2}}$ implique $q \geq T_{m+3}$ et, donc $q<T_{m+3}$ implique $\left\||q \xi \|| \geq c c_{0}^{\frac{1}{2}} T_{m+3}^{-\frac{1}{2}}\right.$. 
Prenons $T_{m+2} \leq q<T_{m+3} ;$ comme $q<T_{m+3}$, on a

$$
\left\||q \xi \|| \geq c c_{0}^{\frac{1}{2}} T_{m+3}^{\frac{-1}{2}} \geq K q^{\frac{-1}{2}}\right.
$$

où $K$ est la constante $c c_{0}\left(c_{1} \beta\right)^{\frac{-1}{2}}$, ce qui donne la conclusion de la proposition.

Ce résultat signifie que $\xi$ est mal approché par les rationnels. En effet, pour tout vecteur $v=\left(\begin{array}{l}v_{1} \\ v_{2}\end{array}\right)$, il existe une suite $\left(q_{n}\right)$ et une constante $K_{0}$ tels que, pour tout $n, q_{n}^{\frac{1}{2}}||\left|q_{n} v\right| \| \leq K_{0}$.

La démonstration élémentaire que nous avons donné de la Proposition 3 est spécifique à l'exemple étudié ; néanmoins, cette proposition est une conséquence d'un théorème classique (cf. Cassels [11]) :

Théorème 4 (Cassels). Soient $x_{1}, \ldots, x_{n}, n$ nombres réels appartenant $\grave{a}$ un corps de nombres totalement réel de degré $n+1$ tel que $1, x_{1}, \ldots, x_{n}$ sont linéairement indépendants sur $\mathbb{Q}$, alors il existe une constante $\gamma$ telle que, pour tout entier naturel $q$ non nul, on ait l'inégalité :

$$
\max _{1 \leq j \leq n}|| q x_{j} \mid \| \geq \gamma q^{-\frac{1}{n}}
$$

Nous montrons, ci-dessous, que la suite $\left(T_{n+3}\right)$ vérifie la propriété

$$
T_{m+3}^{\frac{1}{2}}\left|\left\|T_{m+3} \xi\right\|\right|<c^{\prime} .
$$

En effet

$$
T_{n+3}^{\frac{1}{2}}\left\|T_{n+3} \xi-\begin{array}{c}
T_{n+2} \\
T_{n+1}
\end{array}\right\|=T_{n+3}^{\frac{1}{2}}\left\|\sigma\left(T_{n+3}\right)\right\|=T_{n+3}^{\frac{1}{2}} \theta^{\frac{n}{2}+2} .
$$

La propriété est vérifiée, vu que : $T_{n+3}^{\frac{1}{2}} \theta^{\frac{n}{2}+2} \leq c_{1}^{\frac{1}{2}} \theta^{2}$.

Remarque. Les inégalités (4) et (6) sont vraies avec n'importe quelle norme quitte à modifier les constantes (d'après l'équivalence des normes).

Nous donnons, ci-dessous, une propriété plus forte du vecteur $\xi$ qui exprime, elle aussi, que $\xi$ est mal approché par les rationnels.

Définition 6. Soit $v$ un vecteur de $\mathbb{R}^{2}$ et $\|\cdot\|_{0}$ une norme, on dit que la suite croissante d'entiers $\left(q_{n}\right)$ est la suite de meilleure approximation de $v$ pour la norme $\|\cdot\|_{0} \mathrm{si}$, pour tout $n,\|\| q_{n+1} v\left\|_{0}<\right\| \mid\left\|q_{n} v\right\|_{0}$ et pour tout $q<q_{n+1}, q \neq q_{n+1}$ on a

$$
\left\||| q_{n} v\left|\left\|_{0}<\right\|\right| \mid q v\right\|_{0} .
$$

Proposition 4. Pour toute norme $\|\cdot\|_{0}$, la suite $\left(q_{n}\right)$ des meilleures approximations du vecteur $\xi$ pour $\|.\|_{0}$ vérifie $\left(\frac{q_{n+1}}{q_{n}}\right)$ est bornée. 
Preuve. Supposons $T_{k+2} \leq q_{n}<T_{k+3}$. Pour tout $q<q_{n+1}$, on a $\|q \xi\|_{0} \geq$ $\left\|\mid q_{n} \xi\right\|_{0}$. Or, d'après (4), $\left\|q_{n} \xi\right\|_{0} \geq K q_{n}^{-\frac{1}{2}}>K T_{k+3}^{-\frac{1}{2}}$. D'autre part, pour tout $m$ strictement positif,

$$
\left\|\mid T_{m+k+3} \xi\right\|_{0}<c^{\prime} \theta^{\frac{m}{2}} T_{k+3}^{-\frac{1}{2}}
$$

avec $c^{\prime}=\theta^{2} \frac{c_{1}}{c_{0}^{-\frac{1}{2}}}$.

Comme $\theta<1$, il existe $m_{0}$ tel que $c^{\prime} \theta^{\frac{m_{0}}{2}}<K$, par conséquent on a $\left\|\mid T_{m_{0}+k+3} \xi\right\|\left\|_{0}<\right\|\left\|q_{n} \xi\right\|_{0}$ et $T_{m_{0}+k+3}>q_{n+1}$, donc

$$
\frac{q_{n+1}}{q_{n}}<\frac{T_{m_{0}+k+3}}{T_{k+2}}<\frac{c_{1}}{c_{0}} \beta^{m_{0}+1}=c_{2}
$$

d'où le résultat.

Théorème 5. Pour la norme de Rauzy, la suite de meilleure approximation est la suite $T_{n+3}$.

Pour démontrer ce théorème, on utilise le résultat suivant :

Lemme 4 (Rauzy). (voir [45]) Il existe une constante c $c^{\prime \prime}$ telle que, pour tout $q \in \mathbb{N}$, pour tout $g \in \mathbb{Z}^{2}$,

$$
\|q \xi-g\|<c^{\prime \prime} \quad \text { implique } \delta(q)=q \xi-g .
$$

Soit $n_{0}=8$, alors $\left\|\delta\left(T_{n_{0}+3}\right)\right\|=|\alpha|^{n_{0}+4}<c^{\prime \prime} \approx 0.03$. Comme $|\alpha|<$ 1 , pour tout $n \geq n_{0}$, on a $\left\|\delta\left(T_{n+3}\right)\right\|<c^{\prime \prime}$; par conséquent, pour $q \geq$ $T_{n_{0}+3}$, d'après le Lemme 4 , pour montrer que la suite $\left(T_{n+3}\right)$ est la suite de meilleure approximation, il suffit de vérifier que

$$
\left\|\delta\left(T_{n+3}\right)\right\|<\|\delta(q)\| \quad \text { si } q<T_{n+4} \quad \text { et } \quad q \neq T_{n+3} .
$$

Pour $q<T_{n_{0}+3}=274$, un nombre fini (273) de vérifications permet de conclure, ces vérifications ont été établies par ordinateur.

Lemme 5. Il existe $\Delta$ strictement positif explicite tel que pour toute suite admissible $\epsilon=\left(\epsilon_{n}\right)_{n \geq 0}, \epsilon_{0}=1$,

$$
\Delta(\epsilon)=\left\|\delta(1)+\epsilon_{1} B \delta(1)\right\|-\left\|\sum_{k=2}^{+\infty} \epsilon_{k} B^{k} \delta(1)\right\|>\Delta>0 .
$$

Preuve. 1-er cas. Supposons $\epsilon_{1}=0$, on a

$$
\begin{aligned}
\left\|\sum_{k=2}^{+\infty} \epsilon_{k} B^{k} \delta(1)\right\| & =\left\|B^{2}\left(\sum_{k=0}^{+\infty} \epsilon_{k} B^{k} \delta(1)\right)\right\|=|\alpha|^{2}\left\|\sum_{k=0}^{+\infty} \epsilon_{k} B^{k} \delta(1)\right\| \\
& \leq \frac{|\alpha|^{2}\|\delta(1)\|}{1-|\alpha|^{3}}
\end{aligned}
$$


et donc

$$
\Delta(\epsilon)=\|\delta(1)\|-\frac{|\alpha|^{2}\|\delta(1)\|}{1-|\alpha|^{3}}=\frac{|\alpha|^{4}}{1-|\alpha|^{3}}\left(1-|\alpha|^{2}-|\alpha|^{3}\right)=\Delta_{1}>0 .
$$

2-ème cas. Supposons $\epsilon_{1}=1$ et donc $\epsilon_{2}=0$, on a

$$
\begin{aligned}
\Delta(\epsilon) & =\|\delta(1)+B \delta(1)\|-\left\|\sum_{k=3}^{+\infty} \epsilon_{k} B^{k} \delta(1)\right\| \\
& \geq\|\delta(1)+B \delta(1)\|-\frac{|\alpha|^{7}}{1-|\alpha|^{3}}=\Delta_{2}>0 .
\end{aligned}
$$

On vérifie qu'avec $\Delta_{1} \approx 0,027$ et $\Delta_{2} \approx 0,037, \Delta>|\alpha|^{12} \approx 0,025$.

Soit maintenant $q=\sum_{k \geq 0} \epsilon_{k} T_{k+3}$. Nous supposons que $q<T_{n+4}$ ce qui est équivalent à $\forall k \geq n+1, \epsilon(k)=0$. Nous avons alors $q=\sum_{k=0}^{n} \epsilon_{k} T_{k+3}$, posons $i=\min \left\{k, \epsilon_{k} \neq 0\right\}$, $i$ est différent de $n$ car on suppose que $q \neq T_{n+3}$.

Lemme 6. $S i n-i \geq 8$ alors

$$
\|\delta(q)\|>\left\|\delta\left(T_{n+3}\right)\right\|
$$

Preuve. Grâce au Lemme 5, nous avons

$$
\begin{aligned}
\|\delta(q)\| & =\left\|\sum_{k=i}^{n} \epsilon_{k} B^{k} \delta(1)\right\| \\
& \geq\left\|B^{i} \delta(1)+\epsilon_{i+1} B^{i+1} \delta(1)\right\|-\left\|\sum_{k=i+2}^{n} \epsilon_{k} B^{k} \delta(1)\right\| \\
& \geq|\alpha|^{n+4}=\left\|\delta\left(T_{n+3}\right)\right\| .
\end{aligned}
$$

Si maintenant $n-i \leq 7$

$$
\|\delta(q)\|=\left\|\sum_{k=i}^{n} \epsilon_{k} B^{k} \delta(1)\right\|=|\alpha|^{i}\left\|\sum_{k=0}^{n-i} \epsilon_{k+i} B^{k} \delta(1)\right\| .
$$

On vérifie aisément que $\left\|\sum_{k=0}^{n-i} \epsilon_{k+i} B^{k} \delta(1)\right\| \geq|\alpha|^{n-i+4}$ par un nombre fini de vérifications (37 vérifications) car $n-i \leq 7$, ce qui achève la démonstration.

Le Théorème 5 découle de façon immédiate des lemmes précédents.

\subsection{Calcul de la mesure d'irrationalité. Posons}

$$
K_{0}=\inf \left\{c, \text { il existe un infinité de } q \text { tel que } q^{\frac{1}{2}}\|\| q \xi \|<c\right\}
$$

La constante $K_{0}$ est finie car il existe $K$ tel que, pour tout $q \in \mathbb{N}, q^{\frac{1}{2}}\||q \xi|\|>$ $K$. 
Prenons $T_{n+3} \leq q \leq T_{n+4}$, d'après ce qui précède, $\left\|\left|q \xi\|\| \geq\left\|T_{n+3} \xi\right\|\right|\right.$ implique

$$
q^{\frac{1}{2}}\left|\left\|q \xi\left|\left\|\geq q^{\frac{1}{2}}||\left|T_{n+3} \xi\right|\right\| \geq T_{n+3}^{\frac{1}{2}}\right|\right\| T_{n+3} \xi\|\|=T_{n+3}^{\frac{1}{2}}\left\|\delta\left(T_{n+3}\right)\right\|,\right.
$$

donc

$K_{0}=\inf \left\{c\right.$, il existe un infinité de $n$ tels que $\left.T_{n+3}^{\frac{1}{2}}\left\|\delta\left(T_{n+3}\right)\right\|<c\right\}$

or $\lim _{n \rightarrow+\infty} T_{n+3}^{\frac{1}{2}}\left\|\delta\left(T_{n+3}\right)\right\|=\lim _{n \rightarrow+\infty} T_{n+3}^{\frac{1}{2}} \beta^{n+4}$.

Il est donc clair que

$$
K_{0}=\lim _{n \rightarrow+\infty} T_{n+3}^{\frac{1}{2}}\left\|\delta\left(T_{n+3}\right)\right\|=\frac{1}{\sqrt{\beta^{2}+2 \beta+3}} .
$$

Remarque. On peut montrer, par ces méthodes, que l'ensemble des points d'accumulation de l'ensemble $\left\{T_{n+3}^{\frac{1}{2}} \delta\left(T_{n+3}\right) \mid n \in \mathbb{N}\right\}$ est une ellipse.

\section{Nombres de recouvrement pour la suite de Tribonacci}

Définition 7. Le nombre de recouvrement symbolique $F$ est le plus grand réel $a$ tel que, pour tout entier naturel $h_{0}$, il existe $h \geq h_{0}$, un mot de longueur $h$, noté $w$, et une suite d'indices $i_{n}, n \in \mathbb{N}$, vérifiant

- $w$ apparaît dans $u$ à chaque terme $i_{n}$,

- $i_{n+1}>i_{n}+h-1$ pour tout $n$,

- \#( $\left.\left(i_{n}+m, n \in \mathbb{N}, 0 \leq m \leq h-1\right\} \cap\{0, \ldots, N-1\}\right) \geq a N$ pour tout $N$ assez grand.

Définition 8. Le nombre de recouvrement par cylindres $F_{C}$ est le plus grand réel $a$ tel que, pour tout entier naturel $h_{0}$, il existe $h \geq h_{0}$, un mot de longueur $h$, noté $w$, et une suite d'indices $i_{n}, n \in \mathbb{N}$, vérifiant

- $w$ apparaît dans $u$ à chaque terme $i_{n}$,

- $w$ n'apparaît dans $u$ à aucun autre terme que les $i_{n}$,

- $i_{n+1}>i_{n}+h-1$ pour tout $n$,

- \#(\{in $+m, n \in \mathbb{N}, 0 \leq m \leq h-1\} \cap\{0, \ldots, N-1\}) \geq a N$ pour tout $N$ assez grand.

Ces quantités sont des invariants de conjugaison topologique du système dynamique symbolique associé (voir [12]). Pour les calculer, on utilisera la notion de fréquence sans chevauchement : deux apparitions d'un mot $w$, aux termes $i$ et $j$, sont sans chevauchement si $j>i+h-1$; on pose $d(w)=\lim _{n \rightarrow+\infty} \frac{M_{n}(w)}{n+1}$, où $M_{n}(w)$ est le nombre maximal d'apparitions sans chevauchement de $w$ que l'on peut trouver dans $u_{0} \cdots u_{n}$ (cette limite existe par unique ergodicité). Calculer $F$ pour un système symbolique revient à calculer, pour des mots arbitrairement longs, les plus grandes valeurs de la quantité $\tau(w)=l(w) d(w)$; nous appelons $\tau(w)$ le pavage du mot 
$w$. Calculer $F_{C}$ revient à considérer $\tau(w)$ pour des mots dont toutes les apparitions sont sans chevauchement.

Proposition 5. Pour la suite de Tribonacci

$$
F_{C}=\frac{\beta^{2}+\beta+1}{\beta^{2}+2 \beta+3}=0,618421 \ldots
$$

Preuve. Si, pour $h$, il y a un éclatement, la branche centrale est de longueur 1 , donc $2 h+1=c^{\prime}+m^{\prime}+l^{\prime}-2$, où $c^{\prime}, m^{\prime}$ et $l^{\prime}$ sont les longueurs des circuits. On a $c^{\prime}<m^{\prime}<l^{\prime}$ et $c^{\prime}+m^{\prime}>l^{\prime}+1$, si on se place pour un $h$ assez grand ; donc $h>l^{\prime}-1>m^{\prime}-1$.

Juste après cet éclatement, on passe à $h+1$ et aux longueurs $c=l^{\prime}$, $m=l^{\prime}+c^{\prime}$ et $l=l^{\prime}+m^{\prime} ;$ on a $h+1>c$, et $l \geq m \geq m^{\prime}+c^{\prime} \geq h+1$.

On se place après l'éclatement, et on regarde quels mots peuvent avoir toutes leurs apparitions sans chevauchement. Juste après l'éclatement, la longueur des mots est $h+1$ et celle du circuit court est $c$, et $h+1>c$; ensuite et jusqu'à l'éclatement suivant, la longueur des mots augmente et la longueur du circuit court reste $c$. Si $w$ est sur la branche centrale, il apparaît sur les trois circuits, et chaque apparition sur le circuit court chevauche la suivante car $|w|>c$; on ne peut utiliser un tel mot pour calculer $F_{C}$. Si $w$ est sur la branche courte, ses apparitions sont sans chevauchement car il n'y a jamais deux circuits courts consécutifs ; on a alors un pavage égal à $|w| f_{C}$, qui est maximal juste à l'éclatement suivant, où $|w|=\frac{l+m+c-3}{2}$.

Juste après l'éclatement, la longueur des mots vérifie $h+1 \leq m$, par conséquent, les mots de la branche moyenne apparaissent toujours sans chevauchement ; cette propriété se conserve aux étapes suivantes, jusqu'à ce que la longueur des mots atteigne $m$, ce qui se produit avant l'éclatement suivant, celui-ci ayant lieu quand la longueur des mots vaut $\frac{l+m+c-3}{2} \geq m$. On a donc un pavage possible égal à $m f_{M}, f_{M}$ étant la fréquence du circuit moyen, qui ne change pas avant l'éclatement suivant. Quand la longueur du mot dépasse $m$, on peut améliorer le pavage car après l'éclatement suivant les circuits moyens sont isolés, et le pavage est optimal à l'éclatement, valant $f_{M} \frac{l+m+c-3}{2}$.

Regardons maintenant les apparitions des mots de la branche longue; juste après l'éclatement, comme la longueur des mots vérifie $h+1 \leq$ $l$, les mots appartenant à la branche longue apparaissent toujours sans chevauchement ; cette propriété se conserve aux étapes suivantes, jusqu'à ce que la longueur des mots atteigne $l$, ce qui se produit avant l'éclatement suivant, celui-ci ayant lieu quand la longueur des mots vaut $\frac{l+m+c-3}{2} \geq l$. On a donc un pavage possible égal à $l f_{L}, f_{L}$ étant la fréquence du circuit long, qui ne change pas avant l'éclatement suivant. Quand la longueur du mot dépasse $l$, on ne peut pas améliorer le pavage car, après l'éclatement suivant, il y aura deux circuits longs consécutifs et des apparitions avec chevauchement des mots du circuit long. 
Entre le $n$ - 2-ème et le $n$-1-ème éclatement, $l=T_{n+2}, m=T_{n}+T_{n+1}$, $c=T_{n+1}, f_{L}=\frac{1}{\beta^{n}}, f_{M}=\frac{1}{\beta^{n+1}}, f_{C}=\frac{1}{\beta^{n+2}}$. On vérifie facilement que le meilleur pavage est donc donné par $l f_{L}$, d'où le résultat, sachant que $l f_{L}+m f_{M}+c f_{C}=1$ et que $\frac{T_{n+1}}{T_{n}} \rightarrow \beta$.

La ressemblance entre la valeur de $F_{C}$ et le nombre d'or (qui vaut $1,61803 \ldots$ ) semble purement fortuite. On remarque que $F_{C}$ pour Tribonacci est strictement plus petit que le $F_{C}$ de toute suite sturmienne ([12]).

Proposition 6. Pour la suite de Tribonacci

$$
F=\frac{\beta^{2}+2 \beta+1}{\beta^{2}+2 \beta+3}=0,80122 \ldots .
$$

Preuve. Nous devons estimer la fréquence disjointe d'un mot $w$ : la Proposition 1 nous indique la fréquence des mots, mais aussi, en comparant leur longueur à celle des circuits longs et courts, dans quelle mesure leurs apparitions sont sans chevauchements.

On remarque tout d'abord que, si $h_{1}<h_{2}$ donnent les mêmes fréquences et ont les mêmes rapports avec les longueurs des circuits (c'est-à-dire que les parties entières supérieures de $\frac{h}{\left|L_{h}\right|}, \frac{h}{\left|M_{h}\right|}$ et $\frac{h}{\left|C_{h}\right|}$ ont les mêmes valeurs pour $h=h_{1}$ et $h=h_{2}$ ), la plus grande fréquence sans chevauchement d'un mot de longueur $h_{i}$ est la même pour $i=1$ ou 2 . Le pavage $\tau(w)$ sera donc le meilleur pour $h=h_{2}$; on n'a donc à étudier que les valeurs extrémales de $h$, c'est-à-dire celles correspondant à l'éclatemment et celles pour lesquelles la partie entière supérieure de $\frac{h}{\mid L_{h}}, \frac{h}{\left|M_{h}\right|}$ ou $\frac{h}{\left|C_{h}\right|}$ change de valeur ; en fait, les seules valeurs intéressantes ici sont à l'éclatement, qui est renversant, pour $|w|=m$ et pour $|w|=l$ (en effet, on a toujours $c<|w|<2 m<2 l$, et $|w|=k c$ pour $k>1$ n'apporte rien de nouveau car on ne voit jamais deux circuits $c$ consécutifs).

Pour $h$ fixé, si $w$ est sur la branche courte (resp. longue) du graphe $\Gamma_{h}$, il ne peut apparaître que sur le circuit court (resp. long) et sa fréquence sans chevauchement est inférieure à la fréquence sans chevauchement d'un mot de la branche centrale. En revanche, un mot de la branche centrale apparait à la fois sur le circuit long et le circuit court ; la fréquence et la fréquence sans chevauchement sont donc toujours maximales pour ces derniers. Nous prendrons donc toujours un mot $w$ qui se trouve sur la branche centrale du graphe ; une apparition de $w$ peut se trouver soit sur le circuit court, $C$, de $\Gamma_{h}$, soit sur son circuit moyen, $M$, soit sur son circuit long, $L$.

Pour $|w|=l:|w|>m>c$, et la suite est un enchaînement de circuits $L$, $L C$ et $L M$; le pavage optimal s'obtient en ne gardant que les apparitions de $w$ sur le circuit long, et vaut $l f_{L}$. 
Pour $|w|=m, l>|w|>c$; le pavage optimal s'obtient en ne gardant que les apparitions de $w$ sur le circuit moyen ou long, et vaut $m\left(f_{M}+f_{L}\right)$.

A l'éclatement, $|w|=\frac{l+m+c-3}{2}$, et donc $|w|>l>m>c$ mais $|w| \leq$ $m+c \leq l+c \leq l+m \leq 2 l$. On peut donc sélectionner exactement une apparition sur deux du mot $w$ de la branche centrale, et sa fréquence est $f^{+}=\frac{f_{C}+f_{M}+f_{L}}{2}$. D'où un pavage valant (asymptotiquement) $\frac{1}{4}(c+m+$ $l)\left(f_{C}+f_{L}+f_{M}\right)$.

Le calcul explicite montre que la deuxième formule l'emporte.

On remarque que $F<1$ pour Tribonacci, bien que le système associé soit de rang un, voir Section 8 ci-dessous.

\section{Conséquences ergodiques de l'approximation diophantienne}

Dans tout système mesuré apériodique, pour tout $\epsilon>0$ et tout $h$ arbitrairement grand, on peut trouver (en vertu du Lemme de Rokhlin, voir par exemple [26]) des ensembles disjoints $B, T B, \ldots, T^{h-1} B$ tels que l'union (appelée tour de Rokhlin) de ces ensembles ait une mesure supérieure à $1-\epsilon$. Un système est de rang au plus $r$, si $r$ tours de Rokhlin permettent d'approcher tout ensemble au sens de la mesure, et le nombre de recouvrement est la mesure maximale d'une tour de Rokhlin permettant d'approcher tout ensemble, voir par exemple [21] pour les définitions complètes et un survol de ces notions. Nous nous intéressons à des notions apparentées, lorsque $T$ est la rotation $R_{\alpha}$ sur le tore $\mathbb{T}^{2}$, que l'on sait être de rang un [18], mais nous allons demander à $B$ d'être mieux qu'un ensemble mesurable.

Proposition 7. La rotation $R_{\alpha}$ a un nombre de recouvrement par ensembles simplement connexes d'au moins $\frac{\beta^{2}+\beta+1}{\beta^{2}+2 \beta+3}$; plus précisément, pour tout $h_{0}$, pour tout $a<\frac{\beta^{2}+\beta+1}{\beta^{2}+2 \beta+3}=0,6184 \ldots$, il existe $h \geq h_{0}$ et un ensemble $B$ vérifiant

- $B, R_{\alpha} B, \ldots, R_{\alpha}^{h-1} B$ sont des ensembles simplement connexes disjoints,

- $\mu\left(\cup_{i=0}^{h-1} R_{\alpha}^{i} B\right) \geq a$,

- le diamètre de $B$ est inférieur à $\frac{1}{h_{0}}$.

Preuve. Le calcul de $F_{C}$ pour la suite de Tribonacci se traduit immédiatement, dans le système symbolique $\left(X_{u}, T\right)$, avec les notations ci-dessus, par l'existence d'un cylindre $B^{\prime}$, de hauteur $h$, vérifiant

- $B^{\prime}, T B^{\prime}, \ldots, T^{h-1} B^{\prime}$ sont disjoints,

- $\mu\left(\cup_{i=0}^{h-1} T^{i} B^{\prime}\right) \geq a$.

On prend alors $B=\psi\left(B^{\prime}\right)$ et on sait que les $\psi\left(T^{k} B^{\prime}\right)=R_{\alpha}^{k} B$ sont simplement connexes [45]. Et $B$ est un cylindre de hauteur arbitrairement grande, donc $B^{\prime}$ a un diamètre arbitrairement faible. 
Proposition 8. La rotation $R_{\alpha}$ est de "rang exact par ensembles simplement connexes" au plus égal à trois ; plus précisément, il existe trois suites de cylindres $B_{n, 1}, B_{n, 2}, B_{n, 3}$, trois suites d'entiers $h_{n, 1}, h_{n, 2}, h_{n, 3}$ tendant vers $+\infty$, telles que

- les $R_{\alpha}^{j} B_{n, i}, 1 \leq i \leq 3,1 \leq j \leq h_{n, i}$ sont des ensembles simplement connexes disjoints,

- la réunion des $R_{\alpha}^{j} B_{n, i}, 1 \leq i \leq 3,1 \leq j \leq h_{n, i}$ est de mesure pleine dans $\mathbb{T}^{2}$,

- le diamètre des $B_{n, i}$ tend vers 0 quand $n$ tend vers l'infini.

Preuve. On se place juste après le $n$-ème éclatement ; le graphe $\Gamma_{h}$ n'a de mots que sur trois branches (moyenne, longue et centrale). En raison des propriétés du graphe de Rauzy (voir section 2), on peut alors recouvrir complètement la suite $u$ par des occurences sans chevauchement de trois mots dont les longueurs sont les longueurs des trois branches (voir [4] ; on prend, par exemple, pour chaque branche, le mot formé en concaténant la première lettre de chaque mot de la branche). Cette propriété se traduit par un recouvrement de $X_{u}$ par les itérés de trois cylindres $B_{n, i}^{\prime} ;$ on prend ensuite $B_{n, i}=\psi\left(B_{n, i}^{\prime}\right)$. Comme toutes les longueurs des branches tendent vers l'infini avec $n$, les $B_{n, i}^{\prime}$ sont des cylindres de hauteur arbitrairement grande, donc les $B_{n, i}$ ont un petit diamètre (ils sont de la forme $\alpha^{k} \mathcal{E}+V$, $k$ étant un entier arbitrairement grand et $V$ un vecteur) ; les $R_{\alpha}^{k} B_{n, i}$ sont simplement connexes ; l'union des itérés des $B_{n, i}^{\prime}$ étant exactement $X_{u}$, son image par $\psi$ est un ensemble de mesure pleine.

Notons qu'aucun des deux résultats ci-dessus n'implique l'autre (le second impliquerait le premier avec nombre de recouvrement au moins $\frac{1}{3}$ ). Ces résultats généralisent des résultats sur les rotations de $\mathbb{T}_{1}$ ([19], [12]) et améliorent des résultats de $\mathrm{N}$. Chevallier sur $\mathbb{T}_{2}$ :

Définition 9. Soit $v$ un vecteur de $\mathbb{R}^{2}$, et $\left(q_{n}\right)$ la suite de meilleure approximation de $v$ pour la norme infinie, on dit que $v$ est à quotients partiels bornés si la suite $\left(\frac{q_{n+1}}{q_{n}}\right)$ est bornée ; on dit que $v$ est à quotients partiels non bornés dans le cas contraire.

N. Chevallier [14] a montré que toute rotation de $\mathbb{T}^{2}$ est de rang fini par ensembles convexes. Lorsque le vecteur de la rotation est à quotients partiels non bornés, le résultat est plus précis : la rotation est de rang un par ensembles convexes ; par contre, lorsque le vecteur de la rotation est à quotients partiels bornés, le rang par ensembles convexes est borné par une constante, en général grande, dépendant de $M=\sup _{n}\left(\frac{q_{n+1}}{q_{n}}\right)$. Pour un vecteur donné, on ne sait en général pas si les quotients partiels sont bornés ni quel est l'ordre de grandeur de $M$; pour $R_{\alpha}$, la section 6 nous permet de dire que les quotients partiels sont bornés, avec $M=\sqrt{\beta}$; 
[14] permet alors de borner le rang par ensembles convexes par 3139055, et donc minorer le nombre de recouvrement par convexes par $3 \cdot 10^{-7}$. La Proposition 8 améliore nettement ces bornes, mais en prenant des tours un peu moins "régulières".

\section{Remarques additionnelles}

Les résultats précédents doivent se généraliser aux substitutions :

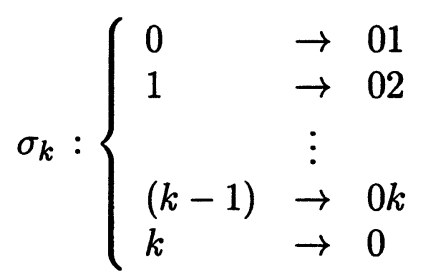

Il serait intéressant de généraliser ces résultats à d'autres systèmes symboliques. Deux familles d'exemples constituent des généralisations naturelles de la substitution de Tribonacci : les suites d'Arnoux-Rauzy et les points fixes de substitution Pisot unitaires.

La représentation géométrique des substitutions Pisot unitaire est un problème difficile. On ne sait, pour l'instant, généraliser aucun des résultats établis dans cet article pour une substitution Pisot unitaire générique.

En ce qui concerne les suites d'Arnoux-Rauzy, l'étude des graphes des mots peut apporter des réponses à certains problèmes combinatoires et ergodiques. Le calcul des invariants $F_{C}$ et $F$ a été effectué par N. Chekhova (cf [12]) pour cette classe d'exemples. Les résultats de ces calculs montrent, en particulier, que les systèmes d'Arnoux-Rauzy ont spectre simple. En ce qui concerne l'aritmétique, les propriétés d'approximation diophantienne, mises en évidence pour le vecteur de la rotation $R_{\alpha}$, semblent spécifiques à l'exemple de la substitution de Tribonacci. Il serait très intéressant de trouver un lien entre les propriétés d'approximation (par exemple le fait que le vecteur de la rotation soit à quotients partiels bornés) et la combinatoire des éclatements d'un système d'Arnoux-Rauzy.

\section{Bibliographie}

[1] P. Alessandri, V. Berthé, Three distances theorems and combinatorics on words. L'Enseignement Mathématique 44 (1998), 103-132.

[2] P. ARNOUX, Un exemple de semi-conjugaison entre un échange d'intervalles et une rotation sur le tore. Bull. Soc. Math. France 116 (1988), 489-500.

[3] P. ARnoux, A. Fisher, The scenery flow for geometric structures on the torus: the linear setting. Chinese Annals of Math., à paraître.

[4] P. ARnoux, G. RAuzy, Représentation géométrique de suites de complexité $2 n+1$. Bull. Soc. Math. France 119 (1991), 101-117.

[5] J. BERSTEL, Recent results in sturmian words. Developments in language theory (Magedburg 1995), World Scientific (96), 13-24.

[6] J. Berstel, M. Pocciola, A geometric proof of the enumeration formula for sturmian words. Internat. J. Algebra Comput. 3 (1993), 349-355. 
[7] V. BERTHÉ, Fréquences des facteurs des suites sturmiennes. Theoret. Comp. Sci. 165 (1996), 295-309.

[8] A. Bertrand-MATHIS, Développement en base $\theta$, répartition modulo un de la suite $\left(x \theta^{n}\right)_{n \geq 0}$, langages codés et $\theta$-shift. Bull. Soc. Math. France 114 (1986), 271-323.

[9] J. CASSAIGNE, Limit values of the reccurence quotient of sturmian sequencies. Theoret. Comp. Sci. 218 (1999), 3-12.

[10] J. Cassaigne, Communication privée.

[11] J. W. S. CASSELS, An introduction to Diophantine approximation. Cambridge Tracts in Mathematics and Mathematical Physics 45, Hafner Publishing Co., New York, 1972. An introduction to diophantine approximation. Cambridge Tracs in Mathematics and Mathematical physics 45 (1972), Cambridge Univ. Press.

[12] N. CHекноva, Covering numbers of rotations. Theoret. Comput. Sci. 230 (2000), 97-116.

[13] N. Cheкhova, Nombres de recouvrement. Thèse de l'Université de la Mediterranée, AixMarseille II, 1997.

[14] N. Chevallier, Meilleures approximations d'un élément du tore $\mathbb{T}^{2}$ et géométrie de la suite des multiples de cet élément. Acta Arithmetica 78 (1996), 19-35.

[15] E. M. Coven, G. A. HedLund, Sequences with minimal block growth. Math. Systems Theory 7 (1973), 138-153.

[16] F. M. Dekking, Recurrent Sets. Adv. Math. 44 (1982), 78-104.

[17] F. M. Dekкing, On the Thue-Morse measure Acta Univ. Carolin. Math. Phys. 33 (1992), 35-40.

[18] A. DEL JUNCO, Transformations with discrete spectrum are stacking transformations. Can. J. Math. 24 (1976), 836-839.

[19] A. DEL Junco, A family of counterexamples in ergodic theory. Israel J. Math. 44 (1983), 160-188.

[20] S. Dulucq, D. Gouyou-Beauchamps, Sur les facteurs des suites de Sturm. Theoret. Comp. Sci. 71 (1990), 381-400.

[21] S. FERENCZI, Systems of finite rank. Colloq. Math. 73 (1997), 35-65.

[22] S. FERENCZI, C. MAUDUIT, Transcendence of numbers with a low complexity expansion. J. Number Theory 47 (1997), 146-161.

[23] A. S. Fraenkel, Systems of numeration. Amer. Math. Monthly 92 (1985), 105-114.

[24] C. Frougny, B. Solomyak, Finite Beta-expansions. Ergodic Theory Dynam. Systems 12 (1992), 713-723.

[25] W. J. GILBERT, Complex numbers with three radix expansions. Can. J. Math. 34 (1982), 1335-1348.

[26] P. Halmos, Lectures on ergodic theory. Publications of the Mathematical Society of Japan 3, The Mathematical Society of Japan, 1956.

[27] G. A. Hedlund, M. Morse, Symbolic dynamics. Amer. J. Math. 60 (1938), 815-866.

[28] G. A. Hedlund, M. Morse, Symbolic dynamics, part II: Sturmian trajectories. Amer. J. Math. 62 (1940) 1-42.

[29] S. Ito, M. Mizutani, Potato Exchange Transformations with Fractal Domains. Preprint.

[30] S. Ito, M. KimurA, On the Rauzy Fractal. Japan J. Indust. Appl. Math. 8 (1991), 461-486.

[31] D. E. KNuth, E. DonAld, The art of computer Programming, Vol 2. Seminumerical algorithms, Second edition. Addison-Wesley Series in Computer Science and Information Processing. Addison-Wesley Publishing Co., Reading, Mass., 1981.

[32] T. Komatsu, On the characteristic word of the inhomogeneous Beatty sequence. Bull. Austr. Math. Soc. 51 (1995), 337-351.

[33] T. KomAtsu, A certain power series associated with a Beatty sequence. Acta Arith. 76 (1996), 109-129.

[34] T. KoMATSU, A certain power series and the inhomogeneous continued fraction expansions. J. Number Th. 59 (1996), 291-312.

[35] T. Komatsu, The fractional part of $n \theta+\phi$ and Beatty sequences. J. Théor. Nombres Bordeaux 7 (1995), 387-406.

[36] A. DE LUCA, F. Mignosi, Some combinatorial properties of sturmian words. Theoret. Comp. Sci. 136 (1994), 361-385. 
[37] A. Messaoudi, Autour du fractal de Rauzy. Thèse de l'Université de la Mediterranée, Aix-Marseille II, 1996.

[38] A. Messaoudi, Propriétés arithmétiques et dynamiques du fractal de Rauzy. J. Théor. Nombres Bordeaux 10 (1998), 135-162.

[39] A. MEssaOUd, Frontière du fractal de Rauzy et système de numération complexe. Acta Arith. 95 (2000), 195-224.

[40] F. Mignosi On the number of factors of Sturmian words. Theoret. Comp. Sci. 82 (1991), 71-84.

[41] B. MossÉ, Notions de reconnaissabilité pour les substitutions et complexité des suites automatiques. Bull. Soc. Math. France 124 (1996), 101-108.

[42] K. NishioKa, J.TAMURA, I. ShIOKAWA, Arithmetical properties of a certain power series. J. Number Th. 42 (1992), 61-87.

[43] B. Praggastis, Markov partitions for hyperbolic toral automorphisms. Ph. D. Thesis, Univ. of Washington, 1992.

[44] M. QUeFFÉLEC, Substitution Dynamical Systems - Spectral Analysis. Lecture Notes in Mathematics 1294, Springer-Verlag, Berlin, 1987.

[45] G. RauzY, Nombres algébriques et substitutions . Bull. Soc. Math. France 110 (1982), 147-178.

[46] G. RAUZY, Suites à termes dans un alphabet fini. Sém. Théor. Nombres Bordeaux (19821983), 1-16.

[47] G. RAUZY, Mots infinis en arithmétique. Automata on infinite words, 165-171, Lecture Notes in Comput. Sci. 192, Springer, Berlin, 1985.

[48] P. SÉÉBold, Fibonacci morphisms and sturmian words. Theoret. Comp. Sci. 88 (1991), $367-384$.

[49] V. SIRVENT, Properties of geometrical realisations of substitutions associated to a family of Pisot numbers. Ph. D. Thesis, Warwick, 1993.

[50] V. SIRVENT, Relationships between the dynamical systems associated to the Rauzy substitutions. Theoret. Comp. Sci. 164 (1996), 41-57.

[51] V. SiRVent, On some dynamical subsets of the Rauzy fractal. Theoret. Comp. Sci. 180 (1997), 363-370.

[52] V. T. Sós, On the distribution mod 1 of the sequence na. Ann. Univ. Sci. Budapest Eötvös Sect. Math. 1 (1958), 127-134.

[53] E. ZECKENDORF, Représentation des nombres naturels par une somme de nombres de Fibonacci ou de nombres de Lucas. Bull. Soc. Roy. Sci. Liège 41 (1972), 179-182.

\section{Nataliya Chekhova}

Université François Rabelais

Département de Mathématiques

Parc de Grandmont, F37200 Tours

France

E-mail : natalya@curie.univ-tours.fr

\section{Ali Messaoudi}

Instituto de matemática

Universidade Federal de Rio do Janeiro

Caixa Postal 68530

21945-970, Rio de Janeiro - RJ

Brazil

E-mail : messaoud@acd.ufrj.br

\section{Pascal Hubert}

Institut de Mathématiques de Luminy

CNRS - UPR 9016 Case 907

163 avenue de Luminy

13288 Marseille Cedex 9

France

E-mail : hubert@iml.univ-mrs.fr 